\title{
Sitagliptin and heart failure hospitalization in patients with type 2 diabetes
}

\author{
Chin-Hsiao Tseng ${ }^{1,2,3}$ \\ ${ }^{1}$ Department of Internal Medicine, National Taiwan University College of Medicine, Taipei, Taiwan \\ 2 Division of Endocrinology and Metabolism, Department of Internal Medicine, National Taiwan University Hospital, Taipei, \\ Taiwan \\ ${ }^{3}$ Division of Environmental Health and Occupational Medicine of the National Health Research Institutes, Zhunan, Taiwan \\ Correspondence to: Chin-Hsiao Tseng, email: ccktsh@ms6.hinet.net
}

Keywords: heart failure, hospitalization, incretin, sitagliptin, Taiwan

Received: April 25, $2016 \quad$ Accepted: June 17, $2016 \quad$ Published: July 09, 2016

\section{ABSTRACT}

This study evaluated the risk of heart failure hospitalization in a 1:1 matched pair sample of sitagliptin ever and never users derived from the Taiwan's National Health Insurance. A total of 85,859 ever users and 85,859 never users matched on 8 digits of propensity score were followed for the first event of heart failure hospitalization until December 31, 2011. The treatment effect (forever versus never users, and for tertiles of cumulative duration of therapy) was estimated by Cox regression incorporated with the inverse probability of treatment weighting using propensity score. Additionally, adjusted hazard ratios for heart failure were estimated for the baseline characteristics in sitagliptin ever users. Results showed that the incidence of heart failure hospitalization was $1,020.16$ and 832.54 per 100,000 personyears, respectively, for ever and never users, with an overall hazard ratio $(95 \%$ confidence intervals) of $1.262(1.167-1.364)$. While compared to never users, the respective hazard ratio for the first, second, and third tertile of cumulative duration $<3.7,3.7-10.3$ and $>10.3$ months was $2.721(2.449-3.023), 1.472(1.318-1.645)$ and 0.515 (0.447-0.594). Older age, longer diabetes duration, male sex, and use of insulin, sulfonylurea, calcium channel blockers, aspirin, ticlopidine, clopidogrel and dipyridamole were significantly associated with a higher risk in sitagliptin users, but dyslipidemia and use of metformin and statin were protective. In conclusion, sitagliptin increases the risk of heart failure hospitalization within one year of its use, but reduces the risk thereafter. Some factors predisposing to sitagliptin-related heart failure are worthy of attention in clinical practice.

\section{INTRODUCTION}

Two clinical trials published in 2013 brought to public concern a possible risk of heart failure associated with the use of dipeptidyl-peptidase 4 (DPP4 ) inhibitors in the treatment of type 2 diabetes mellitus. The Saxagliptin Assessment of Vascular Outcomes Recorded in Patients with Diabetes Mellitus (SAVOR)Thrombolysis in Myocardial Infarction (TIMI) 53 trial (SAVOR-TIMI53) showed an unexpectedly higher risk of heart failure hospitalization in patients treated with saxagliptin $v s$. placebo [3.5\% vs. 2.8\%; hazard ratio 1.27 , $95 \%$ confidence interval $(\mathrm{CI}): 1.07-1.51, P=0.007]$
[1]. Although not significant, more patients treated with alogliptin were diagnosed with heart failure than patients taking placebo, as demonstrated in the Examination of Cardiovascular Outcomes with Alogliptin versus Standard of Care (EXAMINE) [2, 3]. In the meta-analysis by Monami et al. when these two clinical trials were pooled together, the estimated Mantel-Haenszel odds ratio was 1.24 (95\% CI: 1.07-1.45, $P=0.004$ ) [3]. However, such an increased risk of heart failure was not similarly observed in the more recently published Trial Evaluating Cardiovascular Outcomes with Sitagliptin (TECOS), which suggested a neutral risk association between sitagliptin use and placebo, with an estimated hazard ratio 
of 1.00 (95\% CI: $0.83-1.20, P=0.98)$ [4].

Four independent meta-analyses published in 2014 did not make a consistent conclusion. Iqbal et al. estimated a pooled incidence rate ratio $(95 \% \mathrm{CI})$ of $0.55(0.27$ 1.12) for heart failure associated with saxagliptin from 20 clinical trials [5]. Monami et al. estimated a MantelHaenszel odds ratio of 1.19 (95\% CI: $1.03-1.37, P=$ 0.015 ) for DPP-4 inhbitors from 84 randomized trials up to October 1, 2013 [3]. When different DPP-4 inhibitors were estimated separately, the Mantel-Haenszel odds ratio $(95 \% \mathrm{CI})$ was $0.99(0.44-2.24), 0.55(0.20-1.53)$, 1.22 (1.03-1.45), $1.56(0.66-3.65)$ and 1.18 (0.89-1.56), respectively, for sitagliptin, vildagliptin, saxagliptin, linagliptin and alogliptin [3]. Savarese et al. included 94 randomized trials in their meta-analysis and found that long-term (29 weeks or more) use of DPP-4 inhibitors (not specified) was associated with a significantly higher risk of heart failure (relative risk 1.158, 95\% CI: 1.011-1.326, $P=0.034)$, but this was not observed in short-term users (relative risk 0.668, 95\% CI: 0.318-1.400, $P=0.285$ ) [6].

In the fourth meta-analysis, Clifton included 4 cohort studies and 5 randomized trials (including SAVORTIMI53 and EXAMINE) published since October 2013 and estimated an odds ratio of 1.148 (95\% CI: 1.025 $1.287, P=0.017$ ) for DPP-4 inhibitors [7]. When cohort studies and clinical trials were analyzed separately, only the odds ratio derived from the 5 clinical trials was significant (1.239, 95\% CI: $1.078-1.424, P=0.002)$, and that derived from the 4 cohort studies was not $(1.099,95 \%$ CI: 0.913-1.323, $P=0.317$ ) [7].

It is worthy to note that the studies included in the fourth meta-analysis were restricted to recent publications and only one cohort study by Weir et al. was focused on the effect of sitagliptin by using a nested case-control design to analyze the US claims database from a nationally based commercial insurance [8]. They showed that sitagliptin increased the risk of heart failure hospitalization among diabetic patients with pre-existing heart failure $(12.5 \%$ vs. $9.0 \%)$, with an adjusted odds ratio of 1.84 (95\% CI: 1.162.92) [8].

Sitagliptin was the first DPP4 inhibitor approved in Taiwan on July 13, 2007, while the other DPP4 inhibitors (i.e., saxagliptin, vildagliptin and linagliptin) were not approved until after 2009 [9]. A recent study by Wang et al. used a 1:1 matched pairs of users and non-users of sitagliptin in the reimbursement database of the National Health Insurance (NHI) in Taiwan [10]. They showed that the adjusted hazard ratio for the first event of heart failure hospitalization for sitagliptin was 1.21 (95\% CI 1.04-1.42, $P=0.017$ ) [10].

Therefore, whether the most commonly used DPP-4 inhibitor, sitagliptin, may increase the risk of heart failure is under-investigated and inconclusive. While the metaanalysis by Monami et al. [3] including 11 randomized trials suggested a null association, the two analyses of insurance databases showed a significantly higher risk
$[8,10]$. Because not many of these studies evaluated heart failure risk with regards to exposure duration, the present study aimed at evaluating whether sitagliptin use would affect the risk differently among different groups of exposure duration by using the reimbursement database of the NHI. Other incretins were not evaluated because they were not used commonly during the study period. In addition, a new-user design was used to minimize the potential "prevalent user bias" [11]. To reduce the potential risk of "immortal time bias" (the initial period of follow-up during which the outcome can not occur) $[12,13]$, patients included into the study should have been prescribed antidiabetic drugs for at least two times. To avoid the potential confounding from the differences in baseline characteristics associated with treatment allocation in non-random observational studies, a 1:1 matched-pair sample based on 8 digits of propensity score (PS) was used according to the methods described by Parsons [14]. Calculation of standardized difference for each baseline characteristic as recommended by Austin and Stuart [15] was used as a formal test for balance diagnostics. To obtained unbiased estimates, Cox regression models were created by incorporation with the inverse probability of treatment weighting (IPTW) using PS as recommended by Austin [16].

\section{RESULTS}

Table 1 compares the baseline characteristics between ever users and never users of sitagliptin in the matched-pair sample. Although 15 among the 30 variables were significantly different between ever and never users with $P<0.05$, none of the variables had a standardized difference $>10 \%$, suggesting that residual confounding from the baseline characteristics might not be remarkable [15].

Table 2 shows the incidence of heart failure hospitalization by sitagliptin exposure and the hazard ratios comparing sitagliptin exposed to unexposed. During follow-up, a total of 2,988 never users and 1,134 ever users developed first events of heart failure hospitalization, with respective incidence of 832.54 and 1,020.16 per 100,000 person-years. The overall hazard ratio was $1.262(1.167-$ 1.364). For the tertiles of cumulative duration of sitagliptin therapy, significantly increased risk was observed for the first and second tertiles, but the risk was significantly reduced in the third tertiles.

Table 3 shows the adjusted hazard ratios for heart failure hospitalization for all baseline characteristics in patients ever treated with sitagliptin. Older age, longer diabetes duration, male sex, and use of insulin, sulfonylurea, calcium channel blockers, aspirin, ticlopidine, clopidogrel and dipyridamole were significantly associated with a higher risk, but dyslipidemia and use of metformin and statin were associated with a significantly lower risk. 
Table 1: Baseline characteristics of sitagliptin never users and ever users

\begin{tabular}{|c|c|c|c|c|c|c|}
\hline \multirow[t]{3}{*}{ Variables } & \multicolumn{4}{|c|}{ Sitagliptin } & \multirow[t]{3}{*}{$P$} & \multirow{3}{*}{\begin{tabular}{|l}
$\begin{array}{l}\text { Standardized } \\
\text { difference }\end{array}$ \\
\end{tabular}} \\
\hline & \multicolumn{2}{|c|}{$\begin{array}{l}\text { Never users } \\
(n=85859)\end{array}$} & \multicolumn{2}{|c|}{$\begin{array}{l}\text { Ever users } \\
(n=85859)\end{array}$} & & \\
\hline & $n$ & $\%$ & $n$ & $\%$ & & \\
\hline Age (years)* & \multicolumn{2}{|c|}{$54.90 \pm 13.30$} & \multicolumn{2}{|c|}{$54.96 \pm 12.15$} & 0.3214 & 0.43 \\
\hline Diabetes duration (years)* & \multicolumn{2}{|c|}{$3.20 \pm 2.64$} & \multicolumn{2}{|c|}{$3.27 \pm 2.69$} & $<0.0001$ & 2.81 \\
\hline Sex (men) & 47171 & 54.94 & 46687 & 54.38 & 0.0190 & -1.22 \\
\hline Hypertension & 54094 & 63.00 & 54568 & 63.56 & 0.0176 & 1.02 \\
\hline $\begin{array}{l}\text { Chronic obstructive } \\
\text { pulmonary disease }\end{array}$ & 29728 & 34.62 & 30187 & 35.16 & 0.0201 & 1.18 \\
\hline Stroke & 14109 & 16.43 & 14271 & 16.62 & 0.2926 & 0.50 \\
\hline Nephropathy & 11901 & 13.86 & 12067 & 14.05 & 0.2477 & 0.60 \\
\hline Ischemic heart disease & 25359 & 29.54 & 25784 & 30.03 & 0.0249 & 1.09 \\
\hline Peripheral arterial disease & 10892 & 12.69 & 11031 & 12.85 & 0.3148 & 0.49 \\
\hline Obesity & 4434 & 5.16 & 4531 & 5.28 & 0.2927 & 0.48 \\
\hline Dyslipidemia & 53409 & 62.21 & 53775 & 62.63 & 0.0682 & 0.85 \\
\hline Acute pancreatitis & 2248 & 2.62 & 2205 & 2.57 & 0.5138 & -0.32 \\
\hline Alcohol-related diagnoses & 3939 & 4.59 & 3878 & 4.52 & 0.4801 & -0.38 \\
\hline Cancer & 54 & 0.06 & 65 & 0.08 & 0.3131 & 0.47 \\
\hline Sulfonylurea & 60850 & 70.87 & 60309 & 70.24 & 0.0042 & -1.67 \\
\hline Metformin & 60705 & 70.70 & 60099 & 70.00 & 0.0014 & -1.82 \\
\hline Meglitinides & 10284 & 11.98 & 10327 & 12.03 & 0.7495 & 0.11 \\
\hline Acarbose & 13887 & 16.17 & 14292 & 16.65 & 0.0083 & 1.15 \\
\hline Pioglitazone & 5917 & 6.89 & 6599 & 7.69 & $<0.0001$ & 2.93 \\
\hline Rosiglitazone & 11975 & 13.95 & 12513 & 14.57 & 0.0002 & 1.70 \\
\hline Insulin & 9320 & 10.86 & 9023 & 10.51 & 0.0203 & -1.01 \\
\hline Statin & 34256 & 39.90 & 34671 & 40.38 & 0.0410 & 0.90 \\
\hline Fibrate & 23894 & 27.83 & 24267 & 28.26 & 0.0451 & 0.91 \\
\hline $\begin{array}{l}\text { Angiotensin } \\
\text { enzyme inhibitor }\end{array}$ & 34804 & 40.54 & 35156 & 40.95 & 0.0838 & 0.75 \\
\hline Angiotensin receptor blocker & 27222 & 31.71 & 27685 & 32.24 & 0.0166 & 1.08 \\
\hline Calcium channel blocker & 37362 & 43.52 & 37589 & 43.78 & 0.2694 & 0.47 \\
\hline Aspirin & 35529 & 41.38 & 35942 & 41.86 & 0.0432 & 0.95 \\
\hline Ticlopidine & 2079 & 2.42 & 2158 & 2.51 & 0.2191 & 0.60 \\
\hline Clopidogrel & 3336 & 3.89 & 3327 & 3.87 & 0.9105 & -0.04 \\
\hline Dipyridamole & 22728 & 26.47 & 22943 & 26.72 & 0.2403 & 0.56 \\
\hline
\end{tabular}

*Age and diabetes duration are compared by Student's t test and expressed as mean and standard deviation, other variables are compared by Chi square test. 
Table 2: Incidence of heart failure hospitalization by sitagliptin exposure and the hazard ratios comparing sitagliptin exposed to unexposed

\begin{tabular}{|c|c|c|c|c|c|c|}
\hline Sitagliptin use & $\begin{array}{l}\text { Case } \\
\text { number } \\
\text { followed }\end{array}$ & $\begin{array}{l}\text { Incident cases } \\
\text { of heart failure } \\
\text { hospitalization }\end{array}$ & Person-years & $\begin{array}{l}\text { Incidencer rate } \\
\text { of heart failure } \\
\text { hospitalization } \\
\text { (per 100,000 } \\
\text { person-years) }\end{array}$ & $\begin{array}{l}\text { Hazard ratio }(95 \% \\
\text { confidence interval) }\end{array}$ & $P$ \\
\hline Never users & 85859 & 2988 & 358903.58 & 832.54 & 1.000 & \\
\hline Ever users & 85859 & 1134 & 111158.84 & 1020.16 & $1.262(1.167-1.364)$ & $<0.0001$ \\
\hline \multicolumn{7}{|c|}{ Cumulative duration (months) } \\
\hline Never users & 85859 & 2988 & 358903.58 & 832.54 & 1.000 & \\
\hline$<3.7$ & 26643 & 498 & 20635.73 & 2413.29 & $2.721(2.449-3.023)$ & $<0.0001$ \\
\hline $3.7-10.3$ & 30491 & 418 & 35868.65 & 1165.36 & $1.472(1.318-1.645)$ & $<0.0001$ \\
\hline$>10.3$ & 28725 & 218 & 54654.47 & 398.87 & $0.515(0.447-0.594)$ & $<0.0001$ \\
\hline
\end{tabular}

\section{DISCUSSION}

The present study confirmed the findings of an overall increased risk of heart failure hospitalization associated with sitagliptin use as observed in a previous analysis that also used the reimbursement database of the NHI [10]. The estimated overall hazard ratio of 1.262 (95\% CI 1.167-1.364) in the present study (Table 2) was very close to the estimated 1.21 (95\% CI 1.041.42) observed in the previous study [10]. Besides, the present study suggested that such an increased risk was mainly observed during a short duration of its use, i.e., with a cumulative duration of therapy $<1$ year (Table 2). After long-term use, the risk was actually significantly reduced (Table 2). The present study also identified some predictive and some protective factors in association with heart failure hospitalization in sitagliptin users (Table 3). The predictive factors included an older age, a longer diabetes duration, male sex and use of insulin, sulfonylurea, calcium channel blockers and anti-platelet drugs (Table 3). On the other hand, the protective factors included dyslipidemia and use of metformin and statin (Table 3).

A significantly higher risk of heart failure hospitalization associated with short-term rather than long-term use of sitagliptin (Table 2) was contradictory to the finding in the meta-analysis by Savarese et al. [6], which showed a significantly higher risk associated with long-term use of DPP-4 inhibitors for 29 weeks or more. Because this meta-analysis did not consider separate DPP-4 inhibitors, it is not known whether the discrepant findings could be due to the undifferentiation of the DPP-4 inhibitors in the meta-analysis.

The lower risk associated with sitagliptin after a longer duration of its exposure (Table 2) suggested a potentially protective effect of sitagliptin on heart failure. Such a protective effect could be supported by a recent study by dos Santos et al. conducted in human and experimental heart failure [17]. The investigators showed an increase of approximately $130 \%$ in circulating DPP4 activity in patients with heart failure and an inverse correlation between serum DPP-4 activity and left ventricular ejection fraction in patients with heart failure [17]. Furthermore, long-term sitagliptin treatment for 6 weeks significantly improved cardiac performance and mitigated the development and progression of heart failure in rats [17]. Such a beneficial effect of sitagliptin on heart failure could also be demonstrated in another study using pigs as a model [18], but could not be similarly shown when vildagliptin was used in rats [19], suggesting that the protective effect of sitagliptin on heart failure might not be a class effect of DPP-4 inhibitors. However, the observed "protective effect" of sitagliptin after its long-term use may also be explained by reasons not directly related to a real protective effect of the drug on heart failure. First, the reduced risk of heart failure after long-term use might be resulted from the better long-term diabetes control in the users. Second, the lower risk after long-term sitagliptin therapy could be explained by a depletion of susceptible cases. Patients who were predisposed to heart failure might have exhibited the event soon after their use of sitagliptin, leaving the remaining in the cohort of longterm therapy less susceptible to heart failure.

On the other hand, the significantly higher risk of heart failure hospitalization within a short-term exposure to sitagliptin might either be due to the drug per se or due to some other reasons not necessarily indicating a causeeffect relationship. First, the care-givers would be more cautious in the search of severe adverse events when they prescribed a new drug to their patients, leading to a possible detection bias among new users. However, possible detection bias due to the awareness and alertness of heart failure related to the use of DPP-4 inhibitors was less likely because the two clinical trials (i.e., SAVOR- 
Table 3: Adjusted hazard ratios for heart failure hospitalization in patients ever treated with sitagliptin

\begin{tabular}{|c|c|c|c|}
\hline Variable & Interpretation & \begin{tabular}{|l|l|} 
Hazard ratio \\
confidence interval $)$
\end{tabular} & $P$ \\
\hline Age & Every 1-year increment & $1.051(1.045-1.057)$ & $<0.0001$ \\
\hline Diabetes duration & Every 1-year increment & $1.171(1.039-1.320)$ & 0.0098 \\
\hline Sex & Men versus women & $1.033(1.007-1.061)$ & 0.0145 \\
\hline Hypertension & Yes versus no & $0.918(0.761-1.107)$ & 0.3713 \\
\hline Chronic obstructive pulmonary disease & Yes versus no & $1.028(0.910-1.162)$ & 0.6534 \\
\hline Stroke & Yes versus no & $1.009(0.875-1.164)$ & 0.9049 \\
\hline Nephropathy & Yes versus no & $1.276(1.105-1.472)$ & 0.9049 \\
\hline Ischemic heart disease & Yes versus no & $1.093(0.952-1.255)$ & 0.2082 \\
\hline Peripheral arterial disease & Yes versus no & $1.077(0.926-1.252)$ & 0.3348 \\
\hline Obesity & Yes versus no & $1.103(0.820-1.483)$ & 0.5174 \\
\hline Dyslipidemia & Yes versus no & $0.768(0.666-0.885)$ & 0.0003 \\
\hline Acute pancreatitis & Yes versus no & $1.214(0.852-1.729)$ & 0.2827 \\
\hline Alcohol-related diagnoses & Yes versus no & $1.325(1.010-1.739)$ & 0.0419 \\
\hline Cancer & Yes versus no & $2.831(0.703-11.405)$ & 0.1433 \\
\hline Sulfonylurea & Yes versus no & $1.286(1.085-1.526)$ & 0.0038 \\
\hline Metformin & Yes versus no & $0.791(0.678-0.923)$ & 0.0030 \\
\hline Meglitinides & Yes versus no & $1.126(0.958-1.323)$ & 0.1489 \\
\hline Acarbose & Yes versus no & $1.049(0.901-1.220)$ & 0.5386 \\
\hline Pioglitazone & Yes versus no & $1.021(0.832-1.254)$ & 0.8407 \\
\hline Rosiglitazone & Yes versus no & $0.983(0.834-1.158)$ & 0.8386 \\
\hline Insulin & Yes versus no & $1.483(1.264-1.739)$ & $<0.0001$ \\
\hline Statin & Yes versus no & $0.831(0.723-0.955)$ & 0.0089 \\
\hline Fibrate & Yes versus no & $0.963(0.837-1.108)$ & 0.6009 \\
\hline Angiotensin converting enzyme inhibitor & Yes versus no & $1.115(0.964-1.289)$ & 0.1442 \\
\hline Angiotensin receptor blocker & Yes versus no & $1.129(0.986-1.293)$ & 0.0783 \\
\hline Calcium channel blocker & Yes versus no & $1.193(1.020-1.394)$ & 0.0269 \\
\hline Aspirin & Yes versus no & $1.199(1.040-1.383)$ & 0.0125 \\
\hline Ticlopidine & Yes versus no & $1.256(0.981-1.608)$ & 0.0707 \\
\hline Clopidogrel & Yes versus no & $1.468(1.190-1.812)$ & 0.0003 \\
\hline Dipyridamole & Yes versus no & $1.333(1.166-1.525)$ & $<0.0001$ \\
\hline
\end{tabular}

TIMI53 and EXAMINE) reporting an unexpectedly higher risk of heart failure hospitalization related to the use of saxagliptin and alogliptin were published in 2013 $[1,2]$ and the follow-up of the present study cohort was actually ended before this year. Second, although all standardized differences were $<10 \%$ and did not suggest a residual confounding from the differences in the baseline characteristics, 15 out of the 30 variables were significantly different between ever and never users of sitagliptin (Table $1)$. It is worthy to point out that many of the ever users of sitagliptin were characterized by significantly higher prevalence of risk factors of heart failure such as ischemic heart disease, chronic obstructive pulmonary disease, hypertension and use of thiazolidinediones (Table 1). Therefore, it is not known whether residual confounding from these potential risk factors or from some unmeasured risk factors could exert an effect on the observation of a significantly higher risk of heart failure in short-term users of sitagliptin (Table 2).

The discrepant risk association with regards to exposure duration is an interesting finding that has not been reported previously. Estimating an overall risk by lumping together all drug users into an exposure group without considering different durations of exposure would 
not provide full information and might not be adequate in data analyses. Studies mainly including patients within the first year of sitagliptin use may overestimate an overall risk, but studies including more patients with longer duration of exposure might find an attenuated relative risk and concluded with a neutral or even a protective effect.

The discrepant findings between the present study and the recently published TECOS clinical trial which showed a lack of increased risk of heart failure in the sitagliptin group [4] are worthy of discussion. First, the TECOS study is a clinical trial and the generalization of the findings should be limited to patients fitting the inclusion and exclusion criteria set in the trial. On the other hand, the present study mainly reflected a real world scenario. For example, in the TECOS, patients were recruited with a glycated hemoglobin level of $6.5 \%$ to $8.0 \%$ at enrollment [4]. However, in clinical practice in Taiwan, sitagliptin is mainly used as a second or third line therapy because of its higher cost in relative to other preexisting oral antidiabetic drugs. As a result, most patients would be prescribed sitagliptin only when other treatment modalities could not adequately control the blood glucose level, say with a glycated hemoglobin level of $>8.0 \%$. Therefore, the observed outcomes derived from a clinical trial may not be necessarily the same when a medication is widely used in clinical practice in the real world. This discrepancy can also be supported by other observational studies previously conducted in Taiwan [10] and in the USA [8], both reporting a significantly higher risk of heart failure associated with sitagliptin use. Second, ethnicity difference may also partly explain the discrepant findings between the TECOS and the present study. While the present study recruited a more homogeneous group of patients, the TECOS trial was conducted mainly in a mixture of different ethnicities $(67.9 \%$ white, $22.3 \%$ Asian, 3.0\% black and 6.8\% others). It has already been known that DPP-4 inhibitors may have a better efficacy on blood glucose lowering in the Asian populations than in the western people [20], suggesting that the adverse effects related to incretin-based therapy may also show ethnicity difference.

The identification of predictive and protective factors associated with heart failure hospitalization in sitgaliptin users may have some clinical implications. The predictive factors suggested that sitagliptin use should be closely observed for the potential risk of heart failure in patients with an older age, a longer diabetes duration, male sex and use of insulin, sulfonylurea, calcium channel blockers and anti-platelet drugs (Table 3). On the other hand, dyslipidemia and use of metformin and statin might

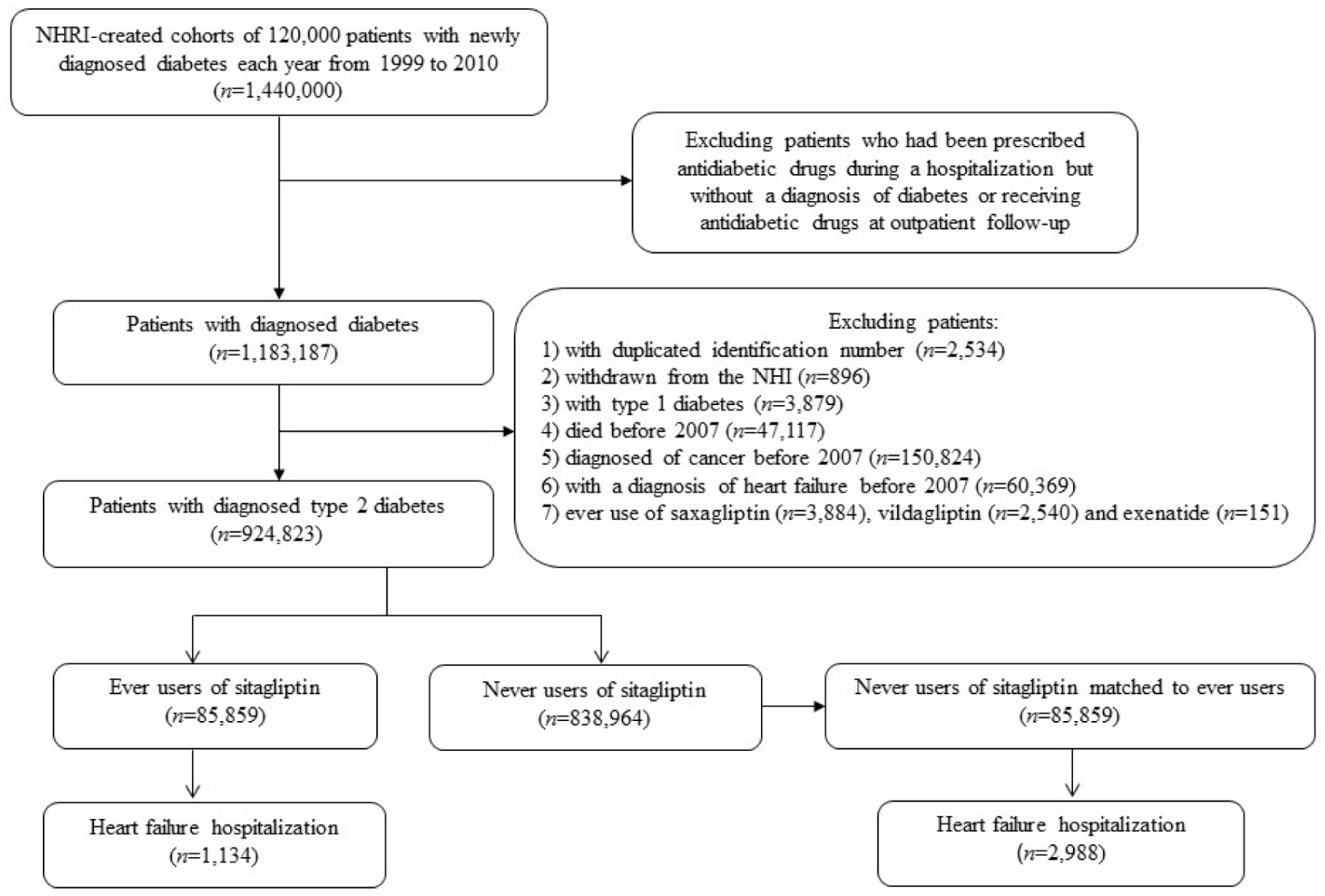

Figure 1: Flowchart showing the procedures followed in creating a cohort of 1:1 matched pair sample of sitagliptin ever and never users from the NHI for the study. NHRI: National Health Research Institutes, NHI: National Health Insurance. 
show a protective effect (Table 3). It is not known whether the lower risk of heart failure associated with dyslipidemia could be due to the protective effect of statin, a lipidlowering drug with well recognized cardiac protective effects commonly used in diabetic patients. Currently it remained difficult to say whether the positive association with some medications and the negative association with others could be due to the direct pharmacological effects of the medications or due to the indications or different disease severities related to their use. More studies are required to clarify the underlying mechanisms or explanations for the relationship with these baseline characteristics.

It is interesting that our previous studies also suggested that sitagliptin may increase the risk of acute pancreatitis [21], pancreatic cancer [22] and thyroid cancer [23] within one or two years of its initiation. The potential risk of these adverse outcomes associated with sitagliptin use have been previously reported and therefore a higher reporting rate might be expected due to clinical awareness and alertness, resulting in a detection bias. However, this could not be applied to the present study because the study period ended by the end of 2011, preceding the publication of most of the reports suggesting a potentially higher risk. There could also be a speculation on the reliability of the database on higher reporting rates of all types of cancer among patients who were initiated with sitagliptin. However, our unpublished analyses did not find such a significantly overall higher risk of other cancers among sitagliptin users, neither within one or two years after its initiation. Therefore, all of these pointed to a true link between sitagliptin use and the adverse outcomes including acute pancreatitis, pancreatic cancer, thyroid cancer and heart failure, especially during the initial period of its use. A similar observation of risk attenuation of these adverse outcomes after a longer duration of sitagliptin use [21-23] might be partly due to the depletion of susceptible cases.

There are some strengths in the study. First, we included all longitudinal data to cover the whole period since the availability of the database in 1996. Second, the large sample size representing the whole nation rendered the generalization of the findings to the whole population in a real world scenario. Third, the use of medical records reduced the bias related to self-reporting.

The limitations include a lack of laboratory data to support the diagnosis of heart failure. However, because the diagnosis of heart failure was made during hospitalization, the reimbursement for such hospitalization should be supported by laboratory data. Second, because no laboratory data were available to define the severity of heart failure, patients hospitalized with such a diagnosis might have represented those with more severe clinical symptoms. Third, the diabetes duration observed from the database may be underestimated because many patients with type 2 diabetes mellitus may remain undiagnosed for several years during the early phase of diabetes. Fourth, we did not have biochemical data such as blood levels of glucose and lipid profiles for evaluating their impacts. Fifth, this study could not evaluate the effects of other DPP4 inhibitors because only the sample size of sitagliptin users was large enough for evaluation.

In conclusions, the present study suggests a biphasic pattern in the association between sitagliptin use and heart failure hospitalization. Users with a cumulative duration $<1$ year may show a significantly higher risk, but a significantly reduced risk can be seen in users with longer duration of exposure. This is the first study pointing out the potential protective effect of sitagliptin on heart failure after long-term use, suggesting that patients who have been using sitagliptin with a cumulative duration $>1$ year should be kept on the medication to be benefited from its potentially protective effect on heart failure. Because spurious association could not be excluded among the short-term users, it remains to be clarified whether the significantly higher risk of heart failure among short-term users can be due to detection bias or residual confounding from the high prevalence of known risk factors of heart failure in the ever users of sitagliptin. The identification of some predictive and some protective baseline characteristics associated with sitagliptin-related heart failure is helpful for the physicians when they prescribe sitagliptin for blood glucose control to their patients.

\section{MATERIALS AND METHODS}

An ethic review board of the National Health Research Institutes (NHRI) approved the study (approval number 99274). The identification information of each patient was scrambled, and written informed consent was not required according to local regulations.

The NHI was implemented since March 1995 and covers more than $99 \%$ of the Taiwanese population, with contracts covering $98 \%$ of the hospitals nationwide. The database keeps detailed records of the insurants' information of principal and secondary diagnostic codes, prescription orders, and claimed expenses from outpatient visits, emergency department visits, and hospital admission.

Figure 1 shows the flowchart for creating a cohort of 1:1 matched pair sample of sitagliptin ever and never users from the NHI. The NHRI created a cohort of 120,000 newly diagnosed diabetic patients in each calendar year for a 12-year period from 1999 to 2010 from the whole nation. The longitudinal reimbursement records of these patients from 1996 to 2011 can be provided for academic research. A patient should not have a diagnosis of diabetes in the previous years when he/she was randomly selected into the cohort for each specific year. The definition of diabetes was based on one of the following two criteria: 1) Diagnosis of diabetes during an admission to the hospital or having been prescribed with antidiabetic drugs during 
hospitalization; or 2) In an outpatient setting within one year, a patient has been diagnosed as having diabetes for two or more times, or diagnosed as having diabetes for one time plus prescribed with antidiabetic drugs for one time. As a result, a total of $1,440,000$ patients with newly diagnosed diabetes were available within these 12 years.

In consideration that some patients might have been given insulin or oral antidiabetic drugs during an admission for some medical conditions but they might not be real cases of diabetes, patients who were recruited based on the criterion of having been prescribed with antidiabetic drugs during hospitalization but had not been followed at the outpatient clinics with a diagnosis of diabetes or had not received antidiabetic drugs at outpatient follow-up were first excluded. This resulted in a sample size of $1,183,187$ patients. Patients who were alive on January 1, 2007 were recruited into the study by the following selection procedures. After exclusion of patients with duplicated identification number $(n=2,534)$, withdrawn from the NHI $(n=896)$, with a diagnosis of type 1 diabetes $(n=3,879)$, died before $2007(n=47,117)$, with a diagnosis of any cancer before $2007(n=150,824)$, with a diagnosis of heart failure before $2007(n=60,369)$, and ever use of saxagliptin $(n=3,884)$, vildagliptin $(n=$ $2,540)$ and exenatide $(n=151$, linagliptin and liraglutide were not available in Taiwan during the study period). As a result, a total of 924,823 patients were available. Among them, 85,859 patients had been newly prescribed with sitagliptin (ever users). To create a 1:1 matched pair sample of 85,859 patients who had not been treated with sitagliptin (never users), the methods described by Parsons based on 8 digits of PS derived from baseline characteristics by logistic regression were used [14]. These methods have also been used in our recently published papers $[23,24]$.

The International Classification of Diseases, Ninth Revision, Clinical Modification (ICD-9-CM) has been used during the study period and diabetes was coded 250.XX. Information of the first event of heart failure hospitalization (ICD-9-CM: 398.91, 402.11, 402.91, 404.11, 404.13, 404.91, 404.93 and 428) was linked from the hospitalization database.

The ICD-9-CM codes for the comorbidities were [25-29]: hypertension 401-405, chronic obstructive pulmonary disease (a surrogate for smoking) 490-496, stroke 430-438, nephropathy 580-589, ischemic heart disease 410-414, peripheral arterial disease 250.7, 785.4, 443.81 and 440-448, obesity 278, dyslipidemia 272.0272.4, acute pancreatitis 577.0, alcohol-related diagnosis 291, 303, 535.3, 571.0-571.3, 980.0, and cancer 140208. Medications included sulfonylurea, metformin, meglitinide, acarbose, pioglitazone, rosiglitazone, insulin, statin, fibrate, angiotensin-converting enzyme inhibitor, angiotensin receptor blocker, calcium channel blocker, aspirin, ticlopidine, clopidogrel, and dipyridamole.

The baseline characteristics of sitagliptin never users and ever users were compared by Student's t test for age and diabetes duration and by Chi-square test for others. The crude incidence density of heart failure hospitalization was calculated for sitagliptin ever users and never users and for the tertiles of cumulative duration of sitagliptin therapy (months). The numerator for the incidence was the number of patients with the first event of heart failure hospitalization during follow-up, and the denominator was the person-years of follow-up. Follow-up started on January 1, 2007 and ended on December 31, 2011, at the time of the first event of heart failure hospitalization, or at the date of the last reimbursement record. In the lack of information on the mortality or migration status of the patients, the last reimbursement record may serve as a surrogate because these patients should be withdrawn from the NHI in Taiwan. Standardized difference for each baseline characteristic was calculated according to the recommendation of Austin and Stuart and a value of $>$ $10 \%$ may indicate meaningful imbalance with potential confounding [15].

The treatment effect was estimated by using PSweighting with the IPTW approach incorporated into a Cox regression [16]. Hazard ratios were estimated forever users versus never users, and for each tertile of cumulative duration of sitagliptin therapy compared to never users as referent.

To identify the predictive and protective characteristics related to sitagliptin-associated heart failure, patients ever treated with sitagliptin were selected for a Cox regression model. In this model heart failure hospitalization was treated as the dependent variable and independent variables included all baseline characteristics.

Analyses were conducted using SAS statistical software, version 9.3 (SAS Institute, Cary, NC). $P<0.05$ was considered statistically significant.

\section{ACKNOWLEDGMENTS}

The study is based in part on data from the National Health Insurance Research Database provided by the Bureau of National Health Insurance, Department of Health and managed by National Health Research Institutes (Registered number 99274). The interpretation and conclusions contained herein do not represent those of Bureau of National Health Insurance, Department of Health or National Health Research Institutes. The author wishes to thank Ms. Ting-Ting Chang for her excellent help in the conduction of statistical analyses.

\section{CONFLICTS OF INTERESTS}

None.

\section{FUNDINGS}

The study was supported by the Ministry of Science 
and Technology (MOST 103-2314-B-002-187-MY3) of Taiwan. The funders had no role in study design, data collection and analysis, decision to publish, or preparation of the manuscript.

\section{REFERENCES}

1. Scirica BM, Bhatt DL, Braunwald E, Steg PG, Davidson J, Hirshberg B, Ohman P, Frederich R, Wiviott SD, Hoffman EB, Cavender MA, Udell JA, Desai NR, et al. SAVORTIMI 53 Steering Committee and Investigators. Saxagliptin and cardiovascular outcomes in patients with type 2 diabetes mellitus. N Engl J Med. 2013;369:1317-26.

2. White WB, Cannon CP, Heller SR, Nissen SE, Bergenstal RM, Bakris GL, Perez AT, Fleck PR, Mehta CR, Kupfer S, Wilson C, Cushman WC, Zannad F; EXAMINE Investigators. Alogliptin after acute coronary syndrome in patients with type 2 diabetes. N Engl J Med. 2013;369:132735.

3. Monami M, Dicembrini I, Mannucci E. Dipeptidyl peptidase-4 inhibitors and heart failure: a meta-analysis of randomized clinical trials. Nutr Metab Cardiovasc Dis. 2014;24:689-97.

4. Green JB, Bethel MA, Armstrong PW, Buse JB, Engel SS, Garg J, Josse R, Kaufman KD, Koglin J, Korn S, Lachin JM, McGuire DK, Pencina MJ, et al. Effect of sitagliptin on cardiovascular outcomes in type 2 diabetes. N Engl J Med. 2015;373:232-42.

5. Iqbal N, Parker A, Frederich R, Donovan M, Hirshberg B. Assessment of the cardiovascular safety of saxagliptin in patients with type 2 diabetes mellitus: pooled analysis of 20 clinical trials. Cardiovasc Diabetol. 2014;13:33.

6. Savarese G, Perrone-Filardi P, D'Amore C, Vitale C, Trimarco B, Pani L, Rosano GM. Cardiovascular effects of dipeptidyl peptidase-4 inhibitors in diabetic patients: A meta-analysis. Int J Cardiol. 2014;181C:239-44.

7. Clifton P. Do Dipeptidyl Peptidase IV (DPP-IV) Inhibitors Cause Heart Failure? Clin Ther. 2014;36:2072-9.

8. Weir DL, McAlister FA, Senthilselvan A, Minhas-Sandhu JK, Eurich DT. Sitagliptin use in patients with diabetes and heart failure: a population-based retrospective cohort study. JACC Heart Fail. 2014;2:573-82.

9. Tseng CH, Lee KY, Tseng FH. An updated review on cancer risk associated with incretin mimetics and enhancers. J Environ Sci Health C Environ Carcinog Ecotoxicol Rev. 2015;33:67-124.

10. Wang KL, Liu CJ, Chao TF, Huang CM, Wu CH, Chen SJ, Yeh CM, Chen TJ, Lin SJ, Chiang CE. Sitagliptin and the risk of hospitalization for heart failure: A population-based study. Int J Cardiol. 2014;177:86-90.

11. Ray WA. Evaluating medication effects outside of clinical trials: new-user designs. Am J Epidemiol. 2003;158:915-20.

12. Stricker BH, Stijnen T. Analysis of individual drug use as a time-varying determinant of exposure in prospective population-based cohort studies. Eur J Epidemiol. 2010;25:245-51.

13. Suissa S. Immortal time bias in pharmaco-epidemiology. Am J Epidemiol. 2008;167:492-9.

14. Parsons LS. Performing a $1: \mathrm{N}$ case-control match on propensity score. http://www.google.com.tw/url?sa $=$ t\&rct $=\mathrm{j} \& \mathrm{q}=\&$ esrc $=\mathrm{s} \&$ source $=$ web\&cd $=1 \& \mathrm{ved}=0 \mathrm{CBs}$ QFjAAahUKEwibi7HllcnIAhUDoJQKHVeZA9A\&url = http\%3A\%2F\%2Fwww2.sas.com\%2Fproceedings\%2Fsug i29\%2F 165-29.pdf\&usg = AFQjCNFOHGWYu8E8Bn4Bo1TUiJKtT987Q (last accessed October 17, 2015).

15. Austin PC, Stuart EA. Moving towards best practice when using inverse probability of treatment weighting (IPTW) using the propensity score to estimate causal treatment effects in observational studies. Stat Med. 2015;34:3661-79.

16. Austin PC. The performance of different propensity score methods for estimating marginal hazard ratios. Stat Med. 2013;32:2837-49.

17. dos Santos L, Salles TA, Arruda-Junior DF, Campos LC, Pereira AC, Barreto AL, Antonio EL, Mansur AJ, Tucci PJ, Krieger JE, Girardi AC. Circulating dipeptidyl peptidase IV activity correlates with cardiac dysfunction in human and experimental heart failure. Circ Heart Fail. 2013;6:1029-38.

18. Gomez N, Touihri K, Matheeussen V, Mendes Da Costa A, Mahmoudabady M, Mathieu M, Baerts L, Peace A, Lybaert P, Scharpé S, De Meester I, Bartunek J, Vanderheyden M, et al. Dipeptidyl peptidase IV inhibition improves cardiorenal function in overpacing-induced heart failure. Eur J Heart Fail. 2012;14:14-21.

19. Yin M, Silljé HH, Meissner M, van Gilst WH, de Boer RA. Early and late effects of the DPP-4 inhibitor vildagliptin in a rat model of post-myocardial infarction heart failure. Cardiovasc Diabetol. 2011;10:85.

20. Cho YM. Incretin physiology and pathophysiology from an Asian perspective. J Diabetes Investig. 2015;6:495-507.

21. Tseng $\mathrm{CH}$. Sitagliptin increases acute pancreatitis risk within 2 years of its initiation: A retrospective cohort analysis of the National Health Insurance database in Taiwan. Ann Med. 2015;47:561-9.

22. Tseng CH. Sitagliptin and pancreatic cancer risk in patients with type 2 diabetes. Eur J Clin Invest. 2016;46:70-9.

23. Tseng $\mathrm{CH}$. Sitagliptin use and thyroid cancer risk in patients with type 2 diabetes. Oncotarget. 2016 Mar 28. doi: 10.18632/oncotarget.8399. [Epub ahead of print].

24. Tseng $\mathrm{CH}$. Metformin may reduce oral cancer risk in patients with type 2 diabetes. Oncotarget. 2016;7:2000-8. doi: 10.18632/oncotarget.6626.

25. Tseng $\mathrm{CH}$. Pioglitazone and bladder cancer: a populationbased study of Taiwanese. Diabetes Care. 2012;35:278-80.

26. Tseng $\mathrm{CH}$. Rosiglitazone may reduce thyroid cancer risk in patients with type 2 diabetes. Ann Med. 2013;45:539-44.

27. Tseng $\mathrm{CH}$. Treatment with human insulin does not increase thyroid cancer risk in patients with type 2 diabetes. Eur J 
Clin Invest. 2014;44:736-42.

28. Tseng $\mathrm{CH}$. Metformin significantly reduces incident prostate cancer risk in Taiwanese men with type 2 diabetes mellitus. Eur J Cancer. 2014;50:2831-7.

29. Tseng $\mathrm{CH}$. Diabetes but not insulin increases the risk of lung cancer: a Taiwanese population-based study. PLoS One. 2014;9:e101553. 